\title{
Quercetin and MicroRNA Interplay in Apoptosis Regulation in Ovarian
}

\section{Cancer}

\author{
Khushbukhat Khan ${ }^{1}$, Zeeshan Javed ${ }^{2, *}$, Haleema Sadia ${ }^{3}$, Javad Sharifi-Rad ${ }^{4, *}$ \\ William C.H. Cho ${ }^{5}$ and Claudio Luparello ${ }^{6}$
}

${ }^{1}$ Atta-ur-Rahman School of Applied Biosciences (ASAB), National University of Sciences and Technology (NUST), Sector H-12, Islamabad 44000, Pakistan

2Office for research innovation and commercialization (ORIC), Lahore Garrison University, Main Campus, Sector C, Phase VI, DHA Lahore Pakistan.

${ }^{3}$ Department of Biotechnology BUITEMS, Quetta, Baluchistan, Pakistan

${ }^{4}$ Phytochemistry Research Center, Shahid Beheshti University of Medical Sciences, Tehran. Iran

${ }^{5}$ Department of Clinical Oncology, Queen Elizabeth Hospital, 30 Gascoigne Road. Hong Kong

${ }^{7}$ Dipartimento di Scienze e Tecnologie Biologiche, Chimiche e Farmaceutiche (STEBICEF), Università di

Palermo, Viale delle Scienze, 90128 Palermo, Italy

Correspondence: zeeshan javed456@yahoo.com (Z.J.); javad.sharifirad@gmail.com(J.S.-R.); 


\begin{abstract}
The multifaceted nature of ovarian cancer has severely hampered the development of effective therapeutics over the years. The composite nature of ovarian cancer makes it therapeutically challenging, therefore, there has been a renewed interest in phytochemistry. Phytochemicals have emerged as a potential therapeutic option due to their limited cytotoxicity and high specificity. Moreover, their signaling inhibition properties have also been studied extensively in recent times. A growing number of data obtained through high-throughput technologies has started to delineate the complex oncogenic signaling networks, thus broadening the therapeutic opportunities. Within such network, microRNAs (miRNAs) have been shown to play a versatile role in the regulation of cancer. Quercetin has been in the spotlight over the years because of its high pharmacological values and substantial evidence has demonstrated its antiproliferative efficacy against various types of cancers. Despite the versatility of quercetin, little is known about its anti-proliferative potential towards ovarian cancer. This review sheds some light on the importance of quercetin as an alternative therapeutic approach to this tumor, furthermore addressing the interplay between miRNAs and quercetin in the regulation of apoptosis in ovarian cancer.
\end{abstract}

Keywords: Apoptosis; Chemo-preventive agent; Ovarian cancer; Phytochemicals; Quercetin; miRNAs. 


\section{Introduction}

Ovarian cancer is a complex disease. Compelling evidence has suggested that specific genetic, epigenetic and environmental factors orchestrate the development of this cancer histotype whose common subtypes are the high grade serous, endometrioid, clear cell, mucinous and low grade serous carcinomas [1,2]. Advanced experimental methodologies have started to dismantle the proteome into sub-classes of proapoptotic and anti-apoptotic proteins. This has led to the nearly complete characterization of tumor necrosis factor (TNF)-related apoptosis inducing ligand (TRAIL) sub-proteomes. Alterations in the TRAIL-mediated signaling pathway are affiliated with the proliferation of ovarian cancer [3]. Considerable efforts have been put to reveal the key biochemical steps involved in apoptosis. This has brought to the limelight that apoptosis is triggered by binding of TNF, TRAIL and Fas ligand. This trimeric complex then facilitates the recruitment of FADD to the death domain (DD) of the receptor [4].

TRAIL binding to the death receptor (DR) initiates the intracellular signaling that can be categorically divided into the extrinsic and intrinsic pathways. The extrinsic signaling is communicated by formation of a death-inducing signaling complex (DISC) which is composed of DR that binds to FADD and pro-caspase 8 [5,7]. Data have suggested the involvement of a protein complex that inhibits TRAIL-mediated signaling via posttranslational modifications. An example may be represented by T3SS effector NleB like protein (NleB1), a protein of a type III secretion system of Escherichia coli, which is involved in the prevention of DISC complex formation via the $\mathrm{N}$-acetylglucosamine transferase-promoted post-translational modification of Arg 117 in the DD of FADD which inhibits the proteolytic activation of caspase-8 [6]. Also, c-FLIP, that possesses two DDs in its N-terminus, is a negative regulator of TRAIL-mediated apoptosis by

preventing the activation of pro-caspase 8 . The intrinsic pathway operates through the caspase- 8 mediated-activation of Bid which, once truncated, moves to mitochondria 
inducing their permeabilization and the resulting release of several pro-apoptotic proteins, such as cytochrome C, apoptosis inducing factor (AIF), Smac/DIABLO, endonuclease $\mathrm{G}$ and Omi/HtrA2 [8].

The induced expression of Smac in reconstituted cells (I DO NOT UNDERSTAND WHAT RECONSTITUtED CELL MEANS AND THERE IS NO RELATED CITATION

IN THIS PARAGRAPH) and Smac mimetics have been reported to enhance apoptosis. Additionally, BH3-only protein family members which include BID, BAD, BIM, NOXA and PUMA also aid in the induction of apoptosis. BH3-only protein and BCL-2 family of protein antagonistically modulate the oligomerization. For instance, BH3-only protein regulates the oligomerization of $\mathrm{BAX}$ and $\mathrm{BAK}$, which are the members of BCL2 family, while anti-apoptotic BCL-2 modulates the BH3-only protein. Both the extrinsic and intrinsic pathways function to induce apoptosis in ovarian cancer. Recent reports have suggested the involvement of a secondary signaling complex in TRAIL-treated cancer cells; consequently, that triggers the activation of downstream effector such as the NF-kB, p38, c-Jun N-terminal kinases (JNK) and extracellular signal-regulated kinases (ERK) (BIBLIOGRAPHY IS NECESSARY IN THE DIFFERENT PARTS OF THIS PARAGRAPH). PROBABLY THIS PARAGRAPH COULD BE DISCARDED OR REWRITTEN

Functional proteomics and phenotypic screening have enabled both molecular biologists and natural product researchers to characterize phytochemicals and their mechanisms of action. There have been radical transformations in our understanding of molecular oncology of ovarian cancer in recent times, as we have begun to unravel its genetic complexity. The significantly high toxicity of chemotherapy, off-target issues and modest clinical outcomes have prompted scientist to analyze various natural products with potential clinical outcomes. 
Quercetin is a bioflavonoid with molecular weight $302.238 \mathrm{~g} / \mathrm{mol}$ and surface area 127 $\mathrm{A}^{2}$, abundantly found in berries, citrus fruits, grapes, onion, lettuces, tomato, broccoli and leeks. Its anti-inflammatory, anti-proliferative, anti-aging, cardio-protective and anti-carcinogenic properties are well documented, and it is regarded as a natural bioactive compound with profound pharmacological implications [14-16]. It has been reported that quercetin modulates the expression of various intracellular pathways such as those controlled by Wnt- $\beta$ catenin, Janus Kinase (JAK) and signal transducer and activator of transcription (STAT), and also vascular endothelial growth factor/vascular endothelial growth factor receptor (VEGF/VEGFR), and epidermal growth factor (EGF)/epidermal growth factor receptor (EGFR) signaling cascades in different types of cancers [9]. This review summarizes the recent development of knowledge on TRAIL, quercetin and their interplay with oncogenic and tumor suppressor microRNAs (miRNAs) involved in mediating apoptosis in ovarian cancer.

\section{Quercetin as an actuator of TRAIL-induced apoptosis}

Cancer cells employ different strategic mechanisms for maintaining growth and avoiding apoptosis. This has fueled much interest in TRAIL-induced intracellular signaling, and a great amount of research has been carried out to unravel the underlying mechanism responsible for resistance against TRAIL-based therapeutics. TRAIL communicates intracellularly via death receptors, the most common of them being DR4 and DR5. Quercetin has shown the ability to stimulate TRAIL-mediated apoptosis in selected cell model systems. As an example, the co-administration of quercetin to resistant breast cancer cells was proven to facilitate TRAIL-mediated apoptosis through induction of c-FLIP degradation and up-regulation of death receptor DR5 [10]. Quercetin induced apoptosis effectively in SMMC-7721, HepG2 and HuH-7 
hepatocellular carcinoma cell lines, which were co-transfected with oncolytic TRAILexpressing ZD55 adenovirus. A reduced expression of I $\mathrm{B} B \alpha$, p65 and p50 components of the NF-kB signalization pathway was also observed when quercetin was administrated in combination with ZD55-TRAIL but not in the presence of ZD55-TRAIL alone, thereby indicating that quercetin was necessary to down-regulate ZD55-TRAILmediated NF- $\mathrm{BB}$ activation and its downstream anti-apoptotic transcriptional targets. A significant reduction in tumor size was observed in mice after the intra-tumor administration of quercetin and ZD55-TRAIL [11]

Quercetin has been reported to sensitize human lymphoma cells to TRAIL-induced apoptosis by inhibiting the expression of survivin and inducing the proteasomal degradation of the anti-apoptotic factor Mcl-1 [Jacquemin G 2012]. Altogether, these findings have prompted scientists to focus on sensitization of resistant cancer cells to TRAIL-based therapeutics. This could mainly be achieved through up-regulation of pro-apoptotic proteins and increasing the expression of death receptor.

\section{Quercetin mediated cell death in ovarian cancer}

In ovarian carcinoma, quercetin is responsible of the inhibition of tumor cell proliferation leading to growth arrest and apoptosis in a dose-dependent manner [17]. Quercetin employs different mechanisms to fulfill its chemo-preventive tasks (figure 1). Several evidence shows that the treatment of ovarian cancer cells with quercetin results in ROS production, elevated expression of pro-apoptotic Bax and Bad factors and caspase-3, along with suppression of apoptosis-preventing Bcl-2 expression [14,18]. Quercetin treatment also induces the generation of ER stress via p53 transcription activation, induction of double-stranded breaks in DNA and delayed repair of damaged DNA [19]. Experimental evidence have proved that exposure to $X$-irradiation along with 
quercetin treatment, exacerbates DNA damage and provokes cell death [20]. Quercetin pre-treatment of OV2008, A2780, and GM9607 ovarian cancer lines was found to resensitize cells to X-irradiation therapy by promoting phosphorylation of ATM, along with enhancing expression of Bax and p21 and facilitating activation of p53 by enhancing its phosphorylation at serines 15 and 20 [19]. In a xenograft model, the cotreatment with radiation and quercetin inhibited tumor growth and promoted apoptosis via activation of p53 and CCAAT enhancer binding protein homologous protein (CHOP) [19]. The role of quercetin in activating the extrinsic apoptotic pathway in ovarian cancer cell via regulating the expression of $\mathrm{CHOP}$ was previously demonstrated [18]. CHOP's contribution in up-regulating DR5 expression in cancers is well-established [21]. As an example, in ovarian cancer SKOV-3 cells quercetin increased the expression of CHOP in a dose-dependent manner, which in turn determined the upregulation of DR5 receptor and sensitization to TRAIL-induced death. On the other hand, in CHOP-silenced SKOV-3 cells, quercetin effect on DR5 production was abrogated. Moreover, treatment with mithramycin A, an inhibitor of the SP1 binding site of DR5 promoter region, abrogated in a dose dependent manner quercetintriggered up-regulation of the death receptor DR5 in the same cell line $[12,13]$. Quercetin stimulation of the apoptosis process was also found to occur in parallel with the inhibition of cellular growth, via blocking the phosphorylation activation of STAT-3 that ultimately induce down-regulation of down-stream Bcl-2 gene expression [22].

Also the apoptotic effect of quercetin in metastatic ovarian cancer was found to be dosedependent. Teekaraman and colleagues [23] treated metastatic ovarian PA-1 cell line with $0-200 \mu \mathrm{M}$ quercetin concentrations for 24 hours and found that the apoptotic influence of quercetin increased with increasing dosage. Among the different doses analyzed via MTT assay, they reported $50 \mu \mathrm{M}$ and $75 \mu \mathrm{M}$ as the most effective against metastatic ovarian cancer. Their findings further revealed that the growth of quercetin- 
treated PA-1 cells was inhibited by the up-regulation of pro-apoptotic elements including, Bid, Bad, Bax, cytochrome c, caspase -3 and 9 and the down-regulation of anti-apoptotic Bcl-xL and Bcl-2 molecules. Thus, quercetin appeared able to promote cellular death in metastatic ovarian cells by activating the intrinsic apoptotic pathway.

A

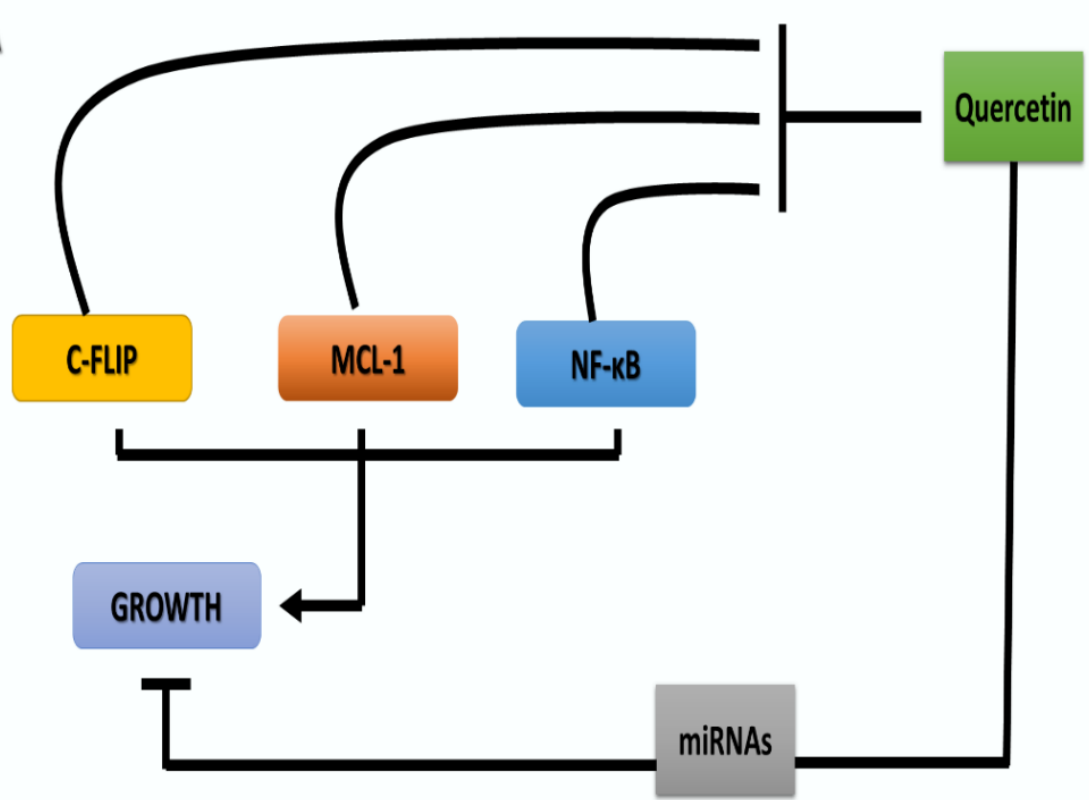

B

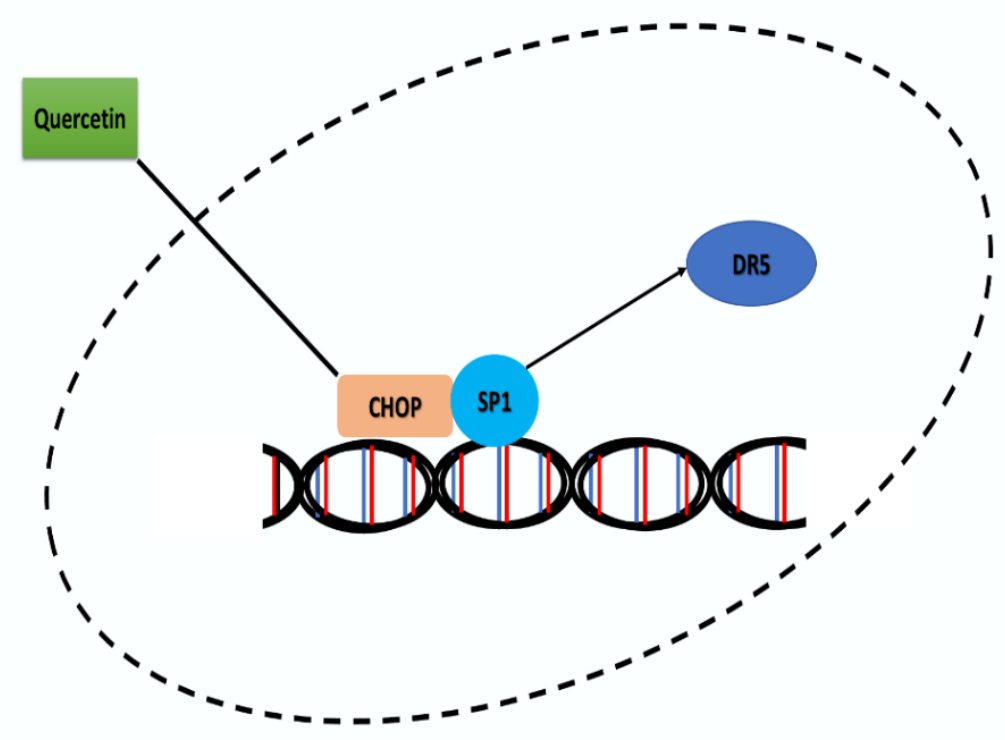

Figure 1: (a) Schematic representation of quercetin mediated regulation of antiapoptotic proteins c-FLIP, MCL-1 and NF-K $\beta$. Quercetin also modulates the expression 
of various oncogenic microRNAs that are involved in suppression of apoptosis and promote proliferation. (b) Quercetin enhances the expression of DR5 necessary for inducing apoptosis via up-regulation of CHOP and SP1.

The efficacy of some selected derivatives of quercetin for curing ovarian cancer is also reported. The therapeutic significance of the monoglucoside of quercetin, isoquercitrin, was analyzed by Michalcova et al. [24] who performed Alamar Blue assay on isoquercitrin treated OVCAR-3 cells and found a negligible effect on cell viability. Similarly, through ELISA and flow cytometry-based apoptosis assays they concluded that isoquercitrin was also unable to induce apoptosis in ovarian cancer cells. However, their findings revealed that at concentrations below $25 \mu \mathrm{g} \mathrm{mL}^{-1}$ this compound prevented intracellular ROS generation, whereas at concentrations above $50 \mu \mathrm{g} \mathrm{mL}^{-1}$ contributed to the generation of oxidative stress, thereby suggesting the benefits of isoquercitrin at lower concentrations against free radical accumulation. Another derivative of quercetin, 3,4',7-O-trimethylquercetin (34'7TMQ), has also been investigated for its anti-cancer activity [25]. In ovarian cancer, 34'7TMQ triggers apoptosis and inhibits cancer cell migration and invasion. Yamauchi et al. [26] demonstrated its cancer suppressive properties in ovarian cancer K-OV-3, CRL11731 and CRL1978 cell lines by analyzing the difference in the expression levels of PCNA, uPA, MMP-2 and PAI-1 through Western blotting. Their results showed that the regulatory influence of $34^{\prime} 7 \mathrm{TMQ}$ varied in the studied cell lines, but in all of them it inhibited metastases by down-regulating the expression of uPA and MMP-2. Ashraf and colleagues [27] further corroborated the therapeutic role of 34'7TMQ in ovarian cancer. They evaluated the expression levels of pro-apoptotic Bax/Bcl-2, caspase 9 and p38 MAPK in 34'7TMQ-treated CRL-1978, CRL-11731 and SK-OV-3 ovarian cancer cell lines via Western blot analysis. They reported that apoptosis was induced in all lines, 
but via a different mechanism since up-regulation of the expression of caspase- 9 was evenly observed in all lines, but the expression levels of Bax/Bcl-2 and p38-MAPK were increased in SK-OV-3 and CRL-1978 cells and in the sole SK-OV-3 cells, respectively. This evidence highlights the novel 34'7TMQ-based therapeutic strategy for ovarian cancer which requires more attention for further unraveling its chemo-preventive properties.

An added advantage of the therapeutic use of quercetin is the lack of negative influences on the function of normal ovarian cells. Kolesarova et al. [28] validated its safety in porcine ovarian granulosa cells and also reported that quercetin was able to promote the release of progesterone and inhibit p53 at $10 \mu \mathrm{mol} \mathrm{L}^{-1}$ concentration, which suggests its dose-dependent effect on the function of ovarian cells. Contrary to these findings, Tarko and colleagues [29] reported that quercetin inhibited the growth of normal porcine ovarian granulosa cells at concentrations higher than $10 \mu \mathrm{gL}^{-1}$ and promoted apoptosis, whereas at concentrations below $10 \mu \mathrm{g} \mathrm{mL}^{-1}$ no effect on cell proliferation could be observed. They also reported that quercetin stimulated the release of oxytocin but did not affect progesterone release. Sirotkin and coworkers [30] further elucidated that exposure of normal ovarian cells to quercetin entailed the downregulation of proliferation-promoting factors such as cyclin B and PCNA at both transcriptional and translational levels and induced the expression of the pro-apoptotic BAX molecule [30]. The outcomes of these studies point towards the inhibitory effect of higher concentrations of quercetin on the normal functionality of healthy ovarian cells, but affirmatively suggest the safety of its use at lower concentrations. Considering the benefits of quercetin in successfully combating ovarian carcinoma at experimental and clinical levels [19,31], it is recommended to devise quercetin-based therapeutics using its lower dosages. In this way, the cytotoxic effect of the drug will be limited to ovarian cancerous cells solely and the damage to healthy cells will be minimized. 
The application of nanocarriers is known to be efficient in constraining quercetin associated cellular death effect to tumor cells and lessening drug-induced functionality damage to healthy ovarian cells. Thermosensitive injectable hydrogel nanocarriers for loading quercetin were designed by utilizing applications of nanotechnology. $\mathrm{Xu}$ et al. [32] constructed a Qu-M-hydrogel composite by encapsulating quercetin into MPEGPCL, followed by its loading in blank thermosensitive hydrogel Pluronic F-127. The MPEG-PCL used contained particles with a minor size of 32nm which had a loading capacity of $7 \%$. They then demonstrated the therapeutic potential of quercetin-loaded nanoparticles in mice model and SKOV-3 cell line. The data obtained indicated that the release of the drug by these hydrogel composites was highly localized and stable in vivo along with its efficient anti-tumor activity. Further, the drug was retained inside the tumor mass for longer time periods which resulted in a high toxicity towards tumor cells with negligible effect on normal cells. Similarly, its growth inhibitory influence and ability for induction of apoptosis was significantly improved in vitro [32]. Additionally, the use of a thermosensitive hydrogel system could assist in overcoming the limitation of poor water solubility of quercetin, its destabilization in physiological media and hence its poor bioavailability [33,34]. Mixed polymeric micelles (MPM) were also tested as nano-carriers for delivery of the quercetin. Patra et al. [35] evaluated the anti-cancer potential of quercetin-containing MPM in SKOV-3 and NCI/ADR cell lines. Because of the stability of MPM in aqueous medium, the drug was retained for longer time periods as compared to free quercetin. Like the Qu-M-hydrogel composite, the release of drug from MPM was slower and this enhanced its cytotoxicity and in far lower dosage the $50 \%$ inhibition of growth ( $\mathrm{IC}_{50}$ value) was achieved [35]. Tiwari and coworkers [36] studied the cytotoxicity of quercetin on PA-1 cells by loading it alone and in combination with gefitinib on polyvinylpyrrolidone-functionalized graphene oxide (GO-PVP). They found that the cytotoxic effect of the combined drugs on PA-1 cells was higher than the single quercetin dose. Moreover, no cytotoxicity was observed on PA-1 
normal cells at concentrations higher than $10 \mu \mathrm{g} / \mathrm{mL}$. They further analyzed the cytotoxicity of GO-PVP-loaded quercetin and its combination with gefitinib on IOSE364 cells and reported that this nanovehicle-based drug system had no significant cytotoxic influence on normal ovarian cells [36]. Hence, their finding was a step forward towards the development of quercetin-based therapeutics, targeted solely to ovarian cancer cells.

\section{Quercetin mediated Regulation of miRNAs in Cancer}

The field of molecular oncology has been revolutionized by the discovery of non-coding RNAs. Recent genomic data has brought to limelight two broad categories of miRNAs, i.e. oncogenic and tumor suppressor miRNAs, both playing diverse roles in cancer cells. Combinations of miRNAs and quercetin have been reported to play a vital role in inducing apoptosis and preventing cell growth (Figure 2). Expression of miRNA let-7c was assessed in PANC-1, ASANPaCa, and AsPC-1 cells. When these cell lines were treated with quercetin, let-7c was shown to be over expressed following exposure. In addition, the Numbl-like protein, that is encoded by the NUMBL gene, was also overexpressed by many folds in let-7 mimic- or quercetin-treated AsPC-1 cells. The Numbl-like protein has been reported to suppress NOTCH signaling pathway, thus preventing cell growth [37]. 


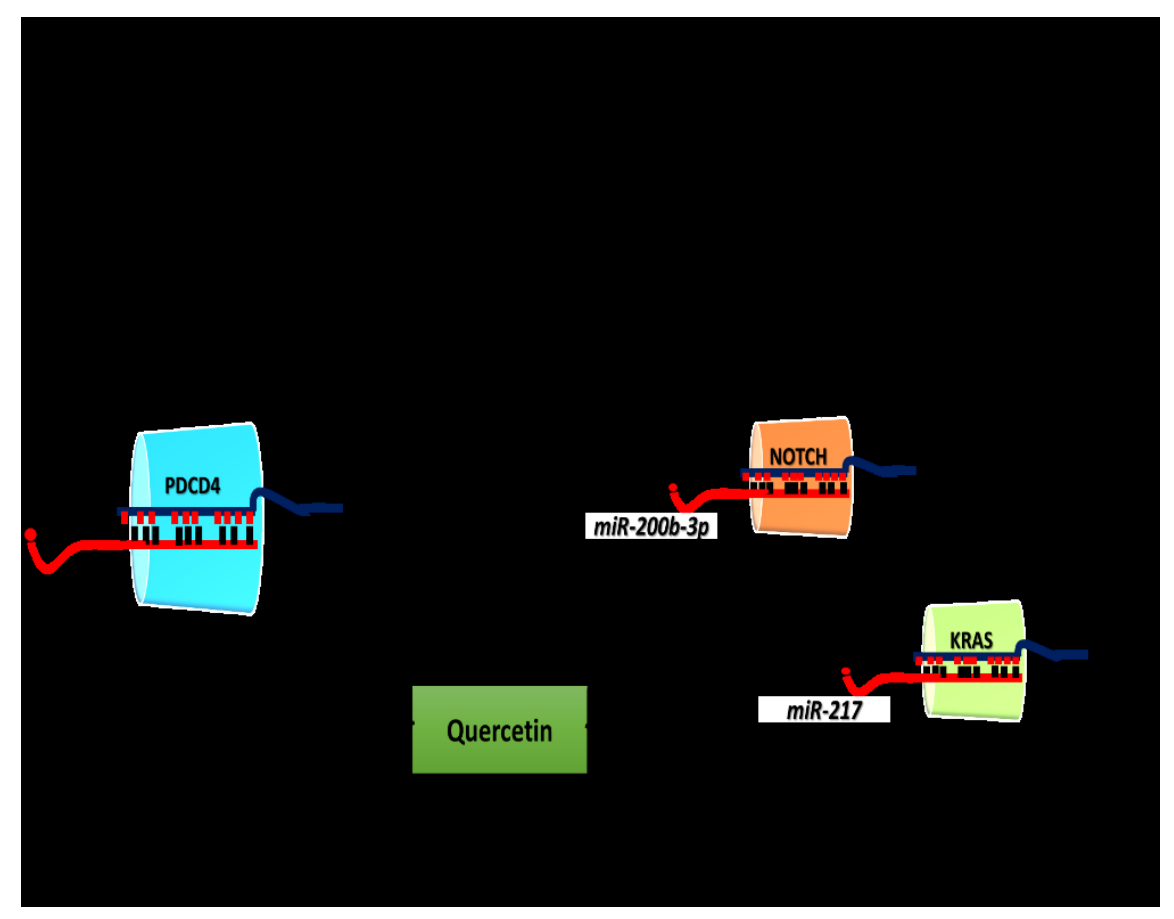

Figure 2: Quercetin interacts with the oncogenic and tumor suppressor miRNAs that regulate the expression of oncogenes as well as apoptosis-inducing genes.

Down-regulation of programmed cell death-4 (PDCD4), up-regulation of miR-21 and malignant transformations were observed in BEAS-2B cells placed under chronic exposure of hexavalent chromium which promoted the formation of ROS. However, administration of quercetin in a dose-dependent manner effectively reversed such effect by up-regulating PDCD4 and reducing miR-21 expression and ROS accumulation in BEAS-2B cells. Significant reduction in malignant transformation of BEAS-2B cells was observed by miR-21 knockdown. Furthermore, in PDCD4-overexpressing BEAS-2B cells, the extent of hexavalent chromium-mediated malignant transformation was considerably decreased [9].

Autophagy related protein 8 (Atg8), a.k.a. GABARAPL1, is known to be associated to the membrane of mature autophagosomes being involved in their biogenesis and also 
in cargo recognition. Its interaction with the microtubule-associated proteins $1 \mathrm{~A}$ and $1 \mathrm{~B}$, light chain-3 (MAP1LC3A), a.k.a. LC3 A, has been confirmed by immunoprecipitation and immunoblotting assays. In gastric cancer, miR-143 targets Atg8 and has been reported to inhibit autophagy. The expression of Atg8 and autophagy markers LC3I/LC3II was reduced by over-expression of miR-143 in MNK28 and AGS cells. However, administration of quercetin to these cell lines restored the expression of these autophagy-associated proteins, thereby demonstrating that the combination of quercetin and miR-143 effectively exerted an anti-cancer effect. Conversely, the treatment of AGS and MNK-28 cells with miRNA mimics suppressed quercetinmediated autophagy [38]. These findings substantiate the role of quercetin in triggering miRNA-based autophagy in gastric cancer.

miR-1275 has been reported to down-regulate the expression of the oncogenic insulinlike growth factor-1 binding protein 1 and 3 (IGF2BP1 and IGFBP3) in Huh hepatocellular carcinoma cells in the presence of quercetin, thus significantly promoting apoptosis in vitro [39].

Quercetin was also proven to up-regulate the expression of miR-200b-3p in AsPC1 pancreatic cancer cells, which, in turn, inhibited NOTCH signaling-mediated selfrenewal [Nwaeburu et al. Mol. Cancer 2017, 16,23]

Therefore, by regulating the expression of various miRNAs in different cancers, quercetin can exert its variegated anti-neoplastic effect. Additional examples may be given. In breast cancer, quercetin was found to promote the expression of miR-146a and inhibit tumor proliferation [40] while by up-regulating miR-217 expression, it was demonstrated that it can confer to osteosarcoma cells the chemosensitivity to cisplatin [41]. Likewise, quercetin was proven to restrain tumor metastasis in oral carcinoma via 
the modulation of miR-16 [42], and to decrease oral cancer cell viability by regulating miR-22 and the downstream WNT1- $\beta$ catenin pathway [43]

\section{MiRNAs modulated by Quercetin in Ovarian Carcinoma}

The crucial role of miRNAs in ovarian tumorigenesis is highlighted by several studies. Through regulation of miRNA expression, tumor cells manage to inhibit cell apoptotic process and ensure their survival. The elevated expression of MiR-23a in ovarian cancer, for instance, was proven to facilitate the inhibition of cellular apoptosis via down-regulation of Bax [44]. Conversely, lower miR-let-7d-5p expression appeared to allow cancer cells to escape apoptosis via modulating p53 signaling pathway, thereby resulting in rapid tumor proliferation along with resistance to chemotherapy [45]. Cisplatin resistance in ovarian carcinoma is realized by increased expression of miR125b which directly targets Bak1 at post-transcriptional level and hinders intrinsic apoptosis [46]. MiR-193 causes induction of apoptosis in epithelial ovarian cancer via up-regulation of caspase-3 and-7 [47]. Bcl-2 like protein 11 (Bim) is targeted by miR-25 in ovarian cancer tissues thus facilitating the suppression of mitochondria-mediated apoptosis [48].

Among the numerous discovered miRNAs, miR-145 contribution in ovarian cancer is of chief importance. Its expression is down-regulated in serum samples, tissues and cell lines of ovarian cancer [49] and restoration of its expression levels this neoplastic histotype was proven to be of therapeutic significance. miR-145 modulates various signaling pathways related to cell growth and apoptosis in ovarian cells. Some of them are enlisted in table 1. Its silencing in ovarian cancer causes the disruption of normalcy in growth rate which leads to tumor development [50]. MiR-145 also facilitates apoptosis induction by modulating the extrinsic apoptotic pathway [51]. Thus, its down-regulation 
further promotes tumor growth and progression by suppression of the pro-apoptotic machinery in ovarian cancer cells $[52,53]$.

Acknowledging the crucial role of miR-145, its silencing serves as a key event in furthering the progression of ovarian carcinogenesis [54]. Hu et al. [55] constructed miR145 mimics and transfected them into SKOV-3 and ES-2 cells, in order to induce miR145 expression. They found that restored miR-145 expression suppressed the proliferation of cancer cells proliferation and prevented their conversion to invasive cells. MiR-145 mimics-treated ovarian cancer cells expressed high levels of Bax protein while expression of Bcl-2 was greatly reduced.

Table 1: List of all targets of miR-145 on ovarian cancer

\begin{tabular}{|c|c|c|c|}
\hline Target & $\begin{array}{l}\text { Modulated cellular } \\
\text { process }\end{array}$ & Comment & Reference \\
\hline $\mathrm{Bcl}-2$ & TRAIL pathway & $\begin{array}{l}\text { Induction of extrinsic } \\
\text { apoptosis. }\end{array}$ & {$[55,56]$} \\
\hline P70S6K1 & mTOR signaling & Downstream target of mTOR. & [49] \\
\hline MUC1 & P53 signaling & $\begin{array}{l}\text { Inhibition of MUC1 via miR- } \\
145 \text { activates p53 signaling and } \\
\text { induces apoptosis. }\end{array}$ & {$[49,57]$} \\
\hline E2F3 & EGFR signaling & $\begin{array}{l}\text { Cell growth inhibition and } \\
\text { increase in apoptosis rate. }\end{array}$ & {$[55,58]$} \\
\hline Metadherin & NF- $\kappa B$ signaling & $\begin{array}{l}\text { miR-145/P53 axis inhibits } \\
\text { metadherin expression. }\end{array}$ & {$[53,59]$} \\
\hline
\end{tabular}




\begin{tabular}{|c|c|c|c|c|}
\hline TRIM 2 & $\begin{array}{l}\text { Intrinsic } \\
\text { pathway }\end{array}$ & apoptotic & $\begin{array}{l}\text { The down-regulation of TRIM } \\
2 \text { induces Bim expression }\end{array}$ & [60] \\
\hline
\end{tabular}

A growing number of evidence has shown the regulatory control of quercetin on miR145 in ovarian cancer. In vitro quercetin treatment of ovarian cancer cells was shown to induce expression of miR-145, and, as demonstrated by Zhou et al. [61], 24 hours after quercetin treatment, proliferation of SKOV-3 and A2780 cells was significantly reduced. They reported the involvement of quercetin-associated up-regulation of miR-145 as the key event behind growth suppression. Mechanistically, the elevated expression of miR145 results in a rise in caspase-3 cleavage, leading to the start of the intrinsic mitochondrial pathway and extrinsic death receptor associated apoptotic pathway [61]. A schematic representation of quercetin regulatory control on miR-145 and induction of apoptosis in ovarian cancer via modulation of different pathways is shown in figure 3. Whether the elevation in caspase-3 cleavage rate is a consequence of a direct miR-145 action or miR-145 may act on some other protein which induces caspase-3 cleavage is so far unclear. However, Hua et al. [55] gave the insight of another process that might happen due to quercetin-induced expression of miR-145. In fact, following the stimulation of miR-145 expression, Bcl-2 expression was negatively modulated and inhibition of cellular growth and proliferation occurred [55] but would the same mechanism also work in quercetin treatment? This is yet to be explored. 


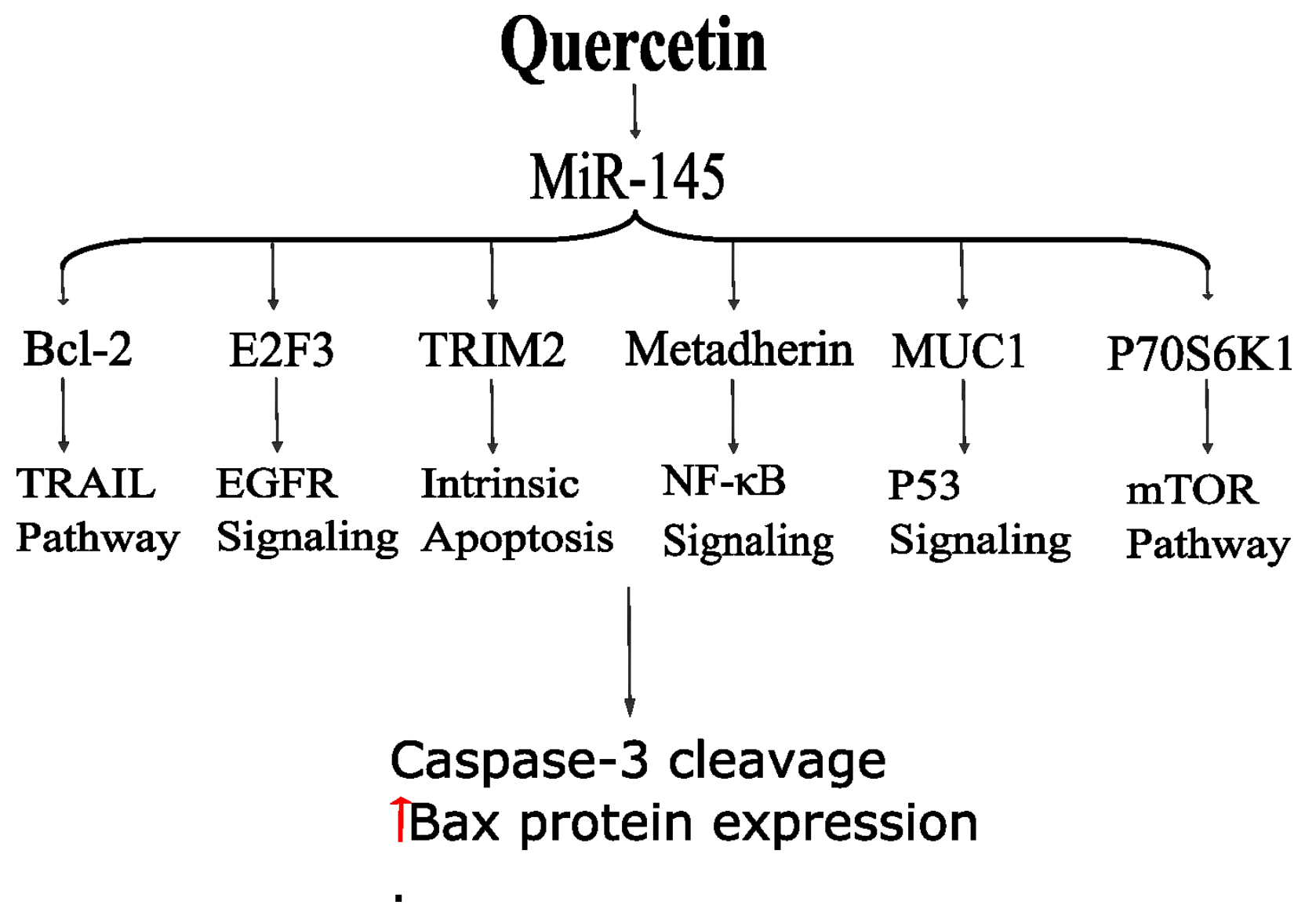

Figure 3: Schematic representation of quercetin effect on miR-145 in ovarian cancer. Quercetin up-regulates the expression of miR-145 which consequently suppresses growth-promoting pathways and initiates extrinsic and intrinsic apoptotic pathway.

\section{Conclusion}

Tumor heterogeneity and deregulated signaling cascades are the two major stumbling blocks that have hampered the efficacy of various therapeutic agents developed under "one gene, one drug" theory. Recent cutting-edge technologies have enabled us to understand the complex genetic framework of various cancers. Thus, it aided us in shifting the paradigm from single-gene/single-drug to a more comprehensive multigene, multi-drug approach. Since cancer is regulated by a plethora of signaling pathways it is indispensable to harvest the maximum potential of quercetin, a 
multiprotein targeting phytochemical. High throughput technologies have enabled us in developing conceptual framework of communication pathways. These developments have provided us with the crystal-clear picture of the core signaling network involved in tumor progression. Quercetin has established itself as a potential apoptosis-inducing agent. Its inclusion into various phases of clinical trials will aid us in analyzing its antitumor potential. Current data has shed light on how quercetin effectively regulates the oncogenic signaling pathway. In xenografted mice, quercetin in combination with TRAIL-based therapeutics successfully inhibited tumor growth. Administration of locked nucleic acid and anti-sense oligonucleotides against oncogenic miRNAs can enhance quercetin-mediated TRAIL activation, consequently maximizing apoptosis in ovarian cancer. The activation of the components of the secondary signaling complex of TRAIL triggers TGF/SMAD transduction cascade. This transduction cascade plays a pivotal role in oncogenesis and metastasis and quercetin has been reported to inhibit the expression of TGF/SMAD transduction cascade via SMADs. The understanding of the mechanism underlying quercetin modulation of the expression of SMAD protein will require further studies.

Author Contributions: All authors contributed to the manuscript. Conceptualization: Z.J., Y.T. and J.S.-R.; Validation investigation, resources, data curation, writing: all authors; review and editing: Z.J., J.S.-R., and C.L. All the authors read and approved the final manuscript.

\section{Funding: None}

Conflicts of Interest: The authors declare no conflict of interest. 


\section{References}

1. Salani, R.; Neuberger, I.; Kurman, R.J.; Bristow, R.E.; Chang, H.-W.; Wang, T.-L.; Shih, I.-M. Expression of extracellular matrix proteins in ovarian serous tumors. International Journal of Gynecological Pathology 2007, 26, 141-146.

2. Farooqi, A.A.; Yaylim, I.; Ozkan, N.E.; Zaman, F.; Halim, T.A.; Chang, H.-W. Restoring TRAIL mediated signaling in ovarian cancer cells. Archivum immunologiae et therapiae experimentalis 2014, 62, 459-474.

3. Bast Jr, R.C.; Hennessy, B.; Mills, G.B. The biology of ovarian cancer: new opportunities for translation. Nature Reviews Cancer 2009, 9, 415.

4. Horak, P.; Pils, D.; Haller, G.; Pribill, I.; Roessler, M.; Tomek, S.; Horvat, R.; Zeillinger, R.; Zielinski, C.; Krainer, M. Contribution of epigenetic silencing of tumor necrosis factor-related apoptosis inducing ligand receptor 1 (DR4) to TRAIL resistance and ovarian cancer. Molecular cancer research 2005, 3, 335-343.

5. Li, S.; Zhang, L.; Yao, Q.; Li, L.; Dong, N.; Rong, J.; Gao, W.; Ding, X.; Sun, L.; Chen, X. Pathogen blocks host death receptor signalling by arginine GlcNAcylation of death domains. Nature 2013, 501, 242.

6. Pearson, J.S.; Giogha, C.; Ong, S.Y.; Kennedy, C.L.; Kelly, M.; Robinson, K.S.; Lung, T.W.F.; Mansell, A.; Riedmaier, P.; Oates, C.V. A type III effector antagonizes death receptor signalling during bacterial gut infection. Nature 2013, 501, 247.

7. Newson, J.P.; Scott, N.E.; Chung, I.Y.W.; Lung, T.W.F.; Giogha, C.; Gan, J.; Wang, N.; Strugnell, R.A.; Brown, N.F.; Cygler, M. Salmonella effectors SseK1 and SseK3 target death domain proteins in the TNF and TRAIL signaling pathways. Molecular \& Cellular Proteomics 2019, 18, 1138-1156.

8. Xu, C.; Liu, X.; Zha, H.; Fan, S.; Zhang, D.; Li, S.; Xiao, W. A pathogen-derived effector modulates host glucose metabolism by arginine GlcNAcylation of HIF-1 $\alpha$ protein. PLoS pathogens 2018, 14, e1007259.

9. Farooqi, A.A.; Jabeen, S.; Attar, R.; Yaylim, I.; Xu, B. Quercetin-mediated regulation of signal transduction cascades and microRNAs: Natural weapon against cancer. Journal of cellular biochemistry 2018, 119, 9664-9674.

10. Manouchehri, J.M.; Turner, K.A.; Kalafatis, M. Trail-induced apoptosis in trail-resistant breast carcinoma through quercetin cotreatment. Breast cancer: basic and clinical research 2018, 12, 1178223417749855.

11. Zou, H.; Zheng, Y.-f.; Ge, W.; Wang, S.-b.; Mou, X.-z. Synergistic Anti-tumour Effects of Quercetin and Oncolytic Adenovirus expressing TRAIL in Human Hepatocellular Carcinoma. Scientific reports 2018, 8, 2182.

12. Li, X.; Wang, X.; Zhang, M.; Li, A.; Sun, Z.; Yu, Q. Quercetin potentiates the antitumor activity of rituximab in diffuse large B-cell lymphoma by inhibiting STAT3 pathway. Cell biochemistry and biophysics 2014, 70, 1357-1362.

13. Kim, J.Y.; Kim, E.H.; Park, S.S.; Lim, J.H.; Kwon, T.K.; Choi, K.S. Quercetin sensitizes human hepatoma cells to TRAlL-induced apoptosis via Sp1-mediated DR5 up-regulation and proteasome-mediated c-FLIPS down-regulation. Journal of cellular biochemistry 2008, 105, 1386-1398. 
14. Shafabakhsh, R.; Asemi, Z. Quercetin: a natural compound for ovarian cancer treatment. Journal of ovarian research 2019, 12, 55.

15. Liu, H.; Lee, J.I.; Ahn, T.-G. Effect of quercetin on the anti-tumor activity of cisplatin in EMT6 breast tumor-bearing mice. Obstetrics \& gynecology science 2019, 62, 242-248.

16. Yang, Y.; Wang, T.; Chen, D.; Ma, Q.; Zheng, Y.; Liao, S.; Wang, Y.; Zhang, J. Quercetin preferentially induces apoptosis in KRAS-mutant colorectal cancer cells via JNK signaling pathways. Cell Biol. Int. 2019, 43, 117-124.

17. Ren, M.X.; Deng, X.H.; Ai, F.; Yuan, G.Y.; Song, H.Y. Effect of quercetin on the proliferation of the human ovarian cancer cell line SKOV-3 in vitro. Experimental and therapeutic medicine 2015, 10, 579-583.

18. Yi, L.; Zongyuan, Y.; Cheng, G.; Lingyun, Z.; GuiLian, Y.; Wei, G. Quercetin enhances apoptotic effect of tumor necrosis factor-related apoptosis-inducing ligand (TRAIL) in ovarian cancer cells through reactive oxygen species (ROS) mediated CCAAT enhancer-binding protein homologous protein (CHOP)-death receptor 5 pathway. Cancer Sci. 2014, 105, 520-527.

19. Gong, C.; Yang, Z.; Zhang, L.; Wang, Y.; Gong, W.; Liu, Y. Quercetin suppresses DNA doublestrand break repair and enhances the radiosensitivity of human ovarian cancer cells via p53dependent endoplasmic reticulum stress pathway. OncoTargets and therapy 2018, 11, 17.

20. Filipa Brito, A.; Ribeiro, M.; Margarida Abrantes, A.; Salome Pires, A.; Jorge Teixo, R.; Guilherme Tralhao, J.; Filomena Botelho, M. Quercetin in cancer treatment, alone or in combination with conventional therapeutics? Curr. Med. Chem. 2015, 22, 3025-3039.

21. Yano, S.; Wu, S.; Sakao, K.; Hou, D.-X. Involvement of ERK1/2-mediated ELK1/CHOP/DR5 pathway in 6-(methylsulfinyl) hexyl isothiocyanate-induced apoptosis of colorectal cancer cells. Biosci., Biotechnol., Biochem. 2019, 83, 960-969.

22. Yang, Z.; Liu, Y.; Liao, J.; Gong, C.; Sun, C.; Zhou, X.; Wei, X.; Zhang, T.; Gao, Q.; Ma, D. Quercetin induces endoplasmic reticulum stress to enhance $c$ DDP cytotoxicity in ovarian cancer: involvement of STAT 3 signaling. The FEBS journal 2015, 282, 1111-1125.

23. Teekaraman, D.; Elayapillai, S.P.; Viswanathan, M.P.; Jagadeesan, A. Quercetin inhibits human metastatic ovarian cancer cell growth and modulates components of the intrinsic apoptotic pathway in PA-1 cell line. Chem.-Biol. Interact. 2019, 300, 91-100.

24. Michalcova, K.; Roychoudhury, S.; Halenar, M.; Tvrda, E.; Kovacikova, E.; Vasicek, J.; Chrenek, P.; Baldovska, S.; Sanislo, L.; Kren, V. In vitro response of human ovarian cancer cells to dietary bioflavonoid isoquercitrin. Journal of Environmental Science and Health, Part B 2019, 1-6.

25. Yamauchi, K.; Mitsunaga, T.; Afroze, S.H.; Uddin, M.N. Structure-Activity Relationships of Methylquercetin on Anti-migration and Anti-proliferation Activity in B16 Melanoma Cells. Anticancer Res. 2017, 37, 1575-1579.

26. Yamauchi, K.; Afroze, S.H.; Mitsunaga, T.; McCormick, T.C.; Kuehl, T.J.; Zawieja, D.C.; Uddin, M.N. 3, 4', 7-O-trimethylquercetin Inhibits Invasion and Migration of Ovarian Cancer Cells. Anticancer Res. 2017, 37, 2823-2829.

27. ASHRAF, A.Z.; Afroze, S.H.; Yamauchi, K.; Zawieja, D.C.; Keuhl, T.J.; Erlandson, L.W.; Uddin, M.N. Differential Mechanism of Action of 3, 4', 7-0-trimethylquercetin in Three Types of Ovarian Cancer Cells. Anticancer Res. 2018, 38, 5131-5137.

28. Kolesarova, A.; Roychoudhury, S.; Klinerova, B.; Packova, D.; Michalcova, K.; Halenar, M.; Kopcekova, J.; Mnahoncakova, E.; Galik, B. Dietary bioflavonoid quercetin modulates porcine ovarian granulosa cell functions in vitro. Journal of Environmental Science and Health, Part B 2019, 1-5.

29. Tarko, A.; Štochmal'ová, A.; Jedličková, K.; Hrabovszká, S.; Vachanová, A.; Harrath, A.H.; Alwasel, S.; Alrezaki, A.; Kotwica, J.; Baláži, A. Effects of benzene, quercetin, and their combination on 
porcine ovarian cell proliferation, apoptosis, and hormone release. Archives Animal Breeding 2019, 62, 345-351.

30. Sirotkin, A.V.; Štochmal'ová, A.; Alexa, R.; Kádasi, A.; Bauer, M.; Grossmann, R.; Alrezaki, A.; Alwasel, S.; Harrath, A.H. Quercetin directly inhibits basal ovarian cell functions and their response to the stimulatory action of FSH. Eur. J. Pharmacol. 2019, 172560.

31. Ferry, D.R.; Smith, A.; Malkhandi, J.; Fyfe, D.W.; deTakats, P.G.; Anderson, D.; Baker, J.; Kerr, D.J. Phase I clinical trial of the flavonoid quercetin: pharmacokinetics and evidence for in vivo tyrosine kinase inhibition. Clin. Cancer. Res. 1996, 2, 659-668.

32. Xu, G.; Li, B.; Wang, T.; Wan, J.; Zhang, Y.; Huang, J.; Shen, Y. Enhancing the anti-ovarian cancer activity of quercetin using a self-assembling micelle and thermosensitive hydrogel drug delivery system. RSC advances 2018, 8, 21229-21242.

33. Cai, X.; Fang, Z.; Dou, J.; Yu, A.; Zhai, G. Bioavailability of quercetin: problems and promises. Curr. Med. Chem. 2013, 20, 2572-2582.

34. Rodriguez, E.B.; Almeda, R.A.; Vidallon, M.L.P.; Reyes, C.T. Enhanced bioactivity and efficient delivery of quercetin through nanoliposomal encapsulation using rice bran phospholipids. J. Sci. Food Agric. 2019, 99, 1980-1989.

35. Patra, A.; Satpathy, S.; Shenoy, A.K.; Bush, J.A.; Kazi, M.; Hussain, M.D. Formulation and evaluation of mixed polymeric micelles of quercetin for treatment of breast, ovarian, and multidrug resistant cancers. International journal of nanomedicine 2018, 13, 2869.

36. Tiwari, H.; Karki, N.; Pal, M.; Basak, S.; Verma, R.K.; Bal, R.; Kandpal, N.D.; Bisht, G.; Sahoo, N.G. Functionalized graphene oxide as a nanocarrier for dual drug delivery applications: The synergistic effect of quercetin and gefitinib against ovarian cancer cells. Colloids Surf. $B$. Biointerfaces 2019, 178, 452-459.

37. Nwaeburu, C.C.; Bauer, N.; Zhao, Z.; Abukiwan, A.; Gladkich, J.; Benner, A.; Herr, I. Up-regulation of microRNA Let-7c by quercetin inhibits pancreatic cancer progression by activation of Numbl. Oncotarget 2016, 7, 58367.

38. Du, F.; Feng, Y.; Fang, J.; Yang, M. MicroRNA-143 enhances chemosensitivity of Quercetin through autophagy inhibition via target GABARAPL1 in gastric cancer cells. Biomedicine \& Pharmacotherapy 2015, 74, 169-177.

39. Fawzy, I.O.; Hamza, M.T.; Hosny, K.A.; Esmat, G.; El Tayebi, H.M.; Abdelaziz, A.I. miR-1275: A single microRNA that targets the three IGF2-mRNA-binding proteins hindering tumor growth in hepatocellular carcinoma. FEBS letters 2015, 589, 2257-2265.

40. Tao, S.-f.; He, H.-f.; Chen, Q. Quercetin inhibits proliferation and invasion acts by up-regulating miR-146a in human breast cancer cells. Mol. Cell. Biochem. 2015, 402, 93-100.

41. Zhang, X.; Guo, Q.; Chen, J.; Chen, Z. Quercetin enhances cisplatin sensitivity of human osteosarcoma cells by modulating microRNA-217-KRAS axis. Molecules and cells 2015, 38, 638.

42. Zhao, J.; Fang, Z.; Zha, Z.; Sun, Q.; Wang, H.; Sun, M.; Qiao, B. Quercetin inhibits cell viability, migration and invasion by regulating miR-16/HOXA10 axis in oral cancer. Eur. J. Pharmacol. 2019, 847, 11-18.

43. Zhang, C.; Hao, Y.; Sun, Y.; Liu, P. Quercetin suppresses the tumorigenesis of oral squamous cell carcinoma by regulating microRNA-22/WNT1/ $\beta$-catenin axis. Journal of Pharmacological Sciences 2019.

44. Zhuang, R.-J.; Bai, X.-X.; Liu, W. MicroRNA-23a depletion promotes apoptosis of ovarian cancer stem cell and inhibits cell migration by targeting DLG2. Cancer biology \& therapy 2019, 20, 897911.

45. Chen, Y.N.; Ren, C.C.; Yang, L.; Nai, M.M.; Xu, Y.M.; Zhang, F.; Liu, Y. MicroRNA let-7d-5p rescues ovarian cancer cell apoptosis and restores chemosensitivity by regulating the p53 signaling pathway via HMGA1. Int. J. Oncol. 2019, 54, 1771-1784. 
46. Kong, F.; Sun, C.; Wang, Z.; Han, L.; Weng, D.; Lu, Y.; Chen, G. miR-125b confers resistance of ovarian cancer cells to cisplatin by targeting pro-apoptotic Bcl-2 antagonist killer 1. Journal of Huazhong University of Science and Technology [Medical Sciences] 2011, 31, 543.

47. Nakano, H.; Yamada, Y.; Miyazawa, T.; Yoshida, T. Gain-of-function microRNA screens identify miR-193a regulating proliferation and apoptosis in epithelial ovarian cancer cells. Int. J. Oncol. 2013, 42, 1875-1882.

48. Zhang, H.; Zuo, Z.; Lu, X.; Wang, L.; Wang, H.; Zhu, Z. MiR-25 regulates apoptosis by targeting Bim in human ovarian cancer. Oncol. Rep. 2012, 27, 594-598.

49. Wu, H.; Xiao, Z.; Wang, K.; Liu, W.; Hao, Q. MiR-145 is downregulated in human ovarian cancer and modulates cell growth and invasion by targeting p70S6K1 and MUC1. Biochem. Biophys. Res. Commun. 2013, 441, 693-700.

50. Moran-Jones, K.; Gloss, B.S.; Murali, R.; Chang, D.K.; Colvin, E.K.; Jones, M.D.; Yuen, S.; Howell, V.M.; Brown, L.M.; Wong, C.W. Connective tissue growth factor as a novel therapeutic target in high grade serous ovarian cancer. Oncotarget 2015, 6, 44551.

51. Li, X.; Chen, W.; Jin, Y.; Xue, R.; Su, J.; Mu, Z.; Li, J.; Jiang, S. miR-142-5p enhances cisplatininduced apoptosis in ovarian cancer cells by targeting multiple anti-apoptotic genes. Biochem. Pharmacol. 2019, 161, 98-112.

52. Zhang, S.; Pei, M.; Li, Z.; Li, H.; Liu, Y.; Li, J. Double-negative feedback interaction between DNA methyltransferase 3A and microRNA-145 in the Warburg effect of ovarian cancer cells. Cancer Sci. 2018, 109, 2734.

53. Dong, R.; Liu, X.; Zhang, Q.; Jiang, Z.; Li, Y.; Wei, Y.; Li, Y.; Yang, Q.; Liu, J.; Wei, J.-J. miR-145 inhibits tumor growth and metastasis by targeting metadherin in high-grade serous ovarian carcinoma. Oncotarget 2014, 5, 10816.

54. Zuberi, M.; Mir, R.; Khan, I.; Javid, J.; Guru, S.; Bhat, M.; Sumi, M.; Ahmad, I.; Masroor, M.; Yadav, P. The promising signatures of circulating microRNA-145 in epithelial ovarian cancer patients. MicroRNA (Shariqah, United Arab Emirates) 2019.

55. Hua, M.; Qin, Y.; Sheng, M.; Cui, X.; Chen, W.; Zhong, J.; Yan, J.; Chen, Y. miR-145 suppresses ovarian cancer progression via modulation of cell growth and invasion by targeting CCND2 and E2F3. Molecular medicine reports 2019, 19, 3575-3583.

56. Fulda, S.; Meyer, E.; Debatin, K.-M. Inhibition of TRAlL-induced apoptosis by Bcl-2 overexpression. Oncogene 2002, 21, 2283.

57. Wei, X.; Xu, H.; Kufe, D. Human MUC1 oncoprotein regulates p53-responsive gene transcription in the genotoxic stress response. Cancer Cell 2005, 7, 167-178.

58. Reimer, D.; Hubalek, M.; Riedle, S.; Skvortsov, S.; Erdel, M.; Concin, N.; Fiegl, H.; Müller-Holzner, E.; Marth, C.; Illmensee, K. E2F3a is critically involved in epidermal growth factor receptordirected proliferation in ovarian cancer. Cancer Res. 2010, 70, 4613-4623.

59. Giopanou, I.; Bravou, V.; Papanastasopoulos, P.; Lilis, I.; Aroukatos, P.; Papachristou, D.; Kounelis, S.; Papadaki, H. Metadherin, p50, and p65 expression in epithelial ovarian neoplasms: an immunohistochemical study. BioMed research international 2014, 2014.

60. Chen, X.; Dong, C.; Law, P.T.; Chan, M.T.; Su, Z.; Wang, S.; Wu, W.K.; Xu, H. MicroRNA-145 targets TRIM2 and exerts tumor-suppressing functions in epithelial ovarian cancer. Gynecologic oncology 2015, 139, 513-519.

61. Zhou, J.; Gong, J.; Ding, C.; Chen, G. Quercetin induces the apoptosis of human ovarian carcinoma cells by upregulating the expression of microRNA-145. Molecular medicine reports 2015, 12, 3127-3131. 